\title{
A review of automation in manufacturing illustrated by a case study on mixed-mode hot forging
}

\author{
Colin S. Harrison* \\ Mechanical and Electrical Engineering, School of Engineering, Glasgow Caledonian University, Cowcaddens Road, \\ Glasgow G4 0BA, UK
}

Received 4 June 2014 / Accepted 8 August 2014

\begin{abstract}
This paper describes a review of automation techniques as currently practiced in the forging and forming industry. That industry has been relatively resistant to handling automation because of the severe challenges that are faced by having typically small batch sizes, extremely challenging operating conditions in terms of temperatures and pressures and lighting conditions. The paper discusses how aspects of these difficulties can be overcome and it is hoped that the specific solutions and methodology presented will find general applications in other aspects of manufacturing industry. The concept of the "Automated Forging of the Future" is introduced in which advanced handling, rapid tooling-change and flexibility, smart metrology, advanced sensing and intelligent gripping techniques are presented. The paper represents the distillation of work on automated forging based on the authors' implementation of a key theme in forging automation in Advanced Forming Research Centre (AFRC), The University of Strathclyde, Glasgow, UK.
\end{abstract}

Key words: Automation, Robotics, Forging, Forming

\section{Introduction}

The manufacturing forging industry can be classified across several industries including Automotive, Aerospace and Medical being dominated by relatively high value added operation, much of the reminder having migrated overseas. Continual cost pressures have meant that industries have had to work smarter rather than harder, since lower costs bases attract plugcompatible manufacturing replication, unless there is a high skill, higher value-add differentiator. Smart automation is presented as one of these differentiators and the ability to quickly change over between batches, achieve higher manufacturing tolerances and overall quality, whilst in many cases increasing volume have been key drivers for the implementation of advanced automation.

Typical product families that have been automated include the production in the automotive industry of gears, hubs, crankshafts with the concurrent objective of optimising materials performance by manipulating materials structure for example, hardness and straight by the automated forging processes themselves.

\footnotetext{
*e-mail: colin.harrison@gcu.ac.uk
}

The key advantages that are presented for automation include:

- Increased Volume (capacity).

- Improved Quality - via consistency of manufacturing and reduction in variability.

- Reduced Costs.

- Reduced dependency on hard to find labour to work in harsh operating conditions.

- Improved Safety Occupational health statistics continue to illustrate that powered presses are a major source of industrial accidents.

However, the financing of these new systems inevitably must and should be filtered by a cost - justification approach in order to test the economic viability of the particular investment proposition. A widely used system is based on manufacturing systems simulation allied to a cost benefit analysis in order to adopt a relatively low cost approach, one the initial business benefits have been outlined. Systems such as Witness [1] Hocus, and the like can be used to explore discrete event simulation approaches, especially where hard production 
data exist on Tact time, time in process, batch sizes and other production data can refine the investment proposition. In industrial situations a question raised almost from the first will always be "What are the benefits" and some attempt at clarifying the financial, engineering and any intangible benefits will need to be constructed with increasing rigour. Some large multinationals have their own specific cost modelling software to address financial returns [2], others use a combination of payback period or internal rate of return, perhaps via a spreadsheet. Intangible benefits can often be influential, but present difficulties in taking them above the line and are usually fought off.

Academic work on forging automation is relatively sparse, however case studies can be found which illustrate the public face of successful implementations [3].

These key drivers automatically define an investment route for scarce capital in that fundamentally the automation engineer is faced with the challenge of automating for lower forecast cost. This means that many developments which might have desirable features are often not cost effective dependant on the analysis regime used. Systems simulation for automation can be applied particularly where the implementation includes data on queuing, mean time between failures (MTBF) and mean time to repair (MTTR) though the results tend to be general in nature. One possible approach when considering a large investment in forging/forming particularly due to the high cost, and longevity of press technology, is often to simulate the process in order to clarify in detail how the modified operation will work and this can then be verified in broad brush terms with a cost modelling approach in order to quantify the benefits.

The existing academic work on "Automation in Forging" is not extensive, for example a search of UK theses on the keywords "Forging Automation" (via the on line index) will reveal a relatively weak result. Contrast this with a search on the abstract keyword "Forging" where over 35 relevant theses will appear including studies on lubrication, wear and damage as well as 3D simulations. Similarly in the main sources (journals having an impact factor and rated conferences) a search using these keywords will result in few results, which are mainly concerned with kinematic modelling $[4,5]$, often without real world data [6], discussions of potential applications [7], thixotropic forging studies [8] and the like. In contrast there has been relatively steady development by suppliers and manufacturers' websites often have useful reporting material, though discussions with robot suppliers (ABB/Fanuc) have revealed that there has been little application development of automation in forging, which reflects the lack of academic output.

This represents an opportunity to fully investigate the integration of automation in forging operations with the intention of cost reduction, increases in quality and consistency and in overall process control.

Forging presents a tremendous challenge to the systems integrator wishing to build in automation to the manufacturing process, combining as it does the effects of varying temperatures, immense potentially destructive forces, and accumulations of hardening lubricant. This combines with the high cost of replacing forging/forming presses where economic lives can be decades, means that retrofitting automatic systems is one feasible industrial approach. Here often the main implementation expertise lies in the area of the industrial supplier or systems integrator. The Forging Industries Association Technology Plan [9] has identified a number of key issues that need to be addressed which prioritises process control and modifications of the "Hoteye" system [10] and other sensing data for Forging.

The methodology of this paper has been to survey the existing, though rather sparse academic literature, interview with industry expert practitioners, and to describe the associated case study. The first task is to try to define what is meant by automation in forging and this description is sufficiently broad that a number of modular topics can be included:

- automated handling,

- automated lubrication,

- automated heating,

- automated process control.

\section{Automation for forging applications}

\subsection{Automated robotic handling}

A key factor in automated handling for forges is the use of robots and the following section places this in context.

The British Automation and Robot Association (BARA) [11] publishes an annual review on robot use based on feedback from 40 leading suppliers [12] which reports that the market is dominated by automotive applications, and recent statistics suggest there are 25,000 robots in service though the international federation suggests that only half of these are less than 12 years old. Even though the market is dominated by automotive applications, trends since 2005 indicate that other sectors are becoming progressively more important. However the use of robotics in forging and is one that is increasing from a relatively low level depending on category definition (see Figure 1) and the non-automotive applications have continued to grow.

It is estimated that $75 \%$ of installed robots are of the 6 axis type (BARA-2009) figures, although these can also be mounted on rails or hang from a track with over $90 \%$ of the 460 installations in 2009 being priced at between $£ 10,000$ and $£ 50,000$ although there is an increasingly active market in robot refurbishment, which on average is about $50 \%$ of the cost of a new installation.

A recent review by Rimrock Corp on forge shop automation $[13,14]$ describes an approach to adopting automation by subdividing the process (of handling) into the following operations:

- billet loading,

- trim press loading,

- parts transfer robot,

- spray robot.

A conventional approach is to apply these to a pre-existing press situation in order to improve the process and the benefits as listed can be substantial though there is cost associated with redesign of the process and part which is often difficult to quantify at the outset though can provide substantial benefits in successful installations. This approach could be described as 


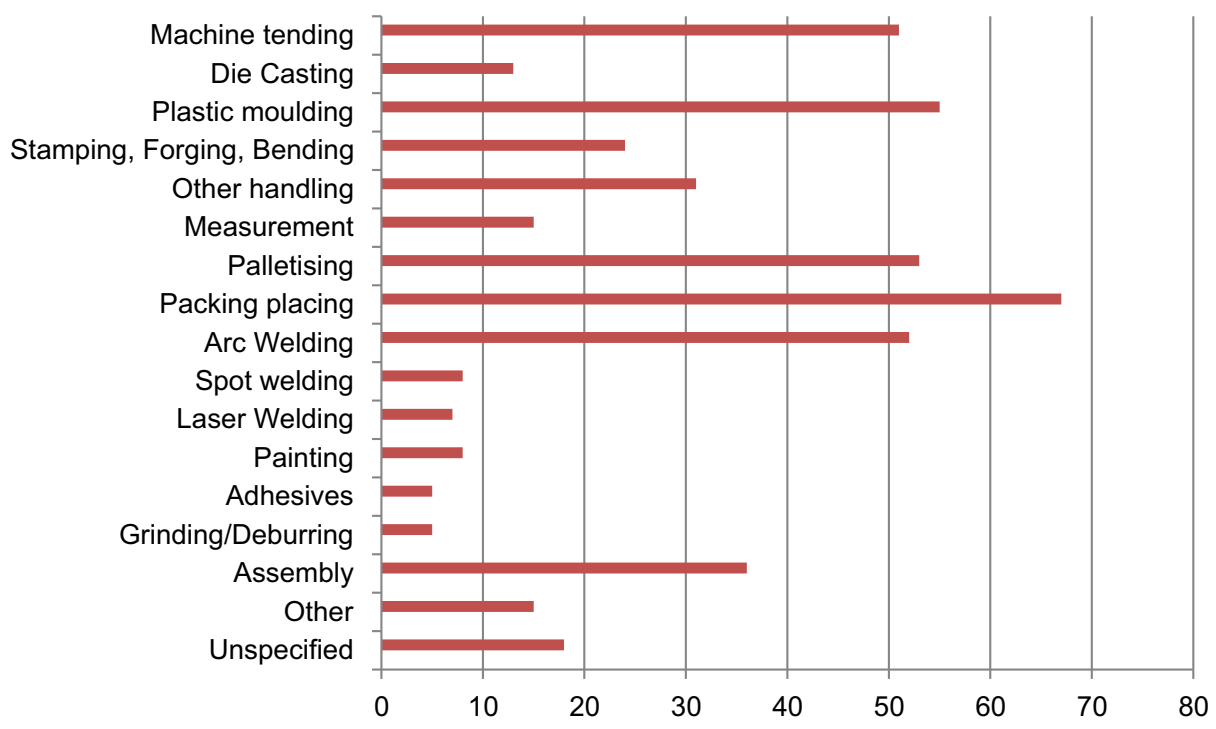

Figure 1. UK Robot application analysis for 2009 (BARA).

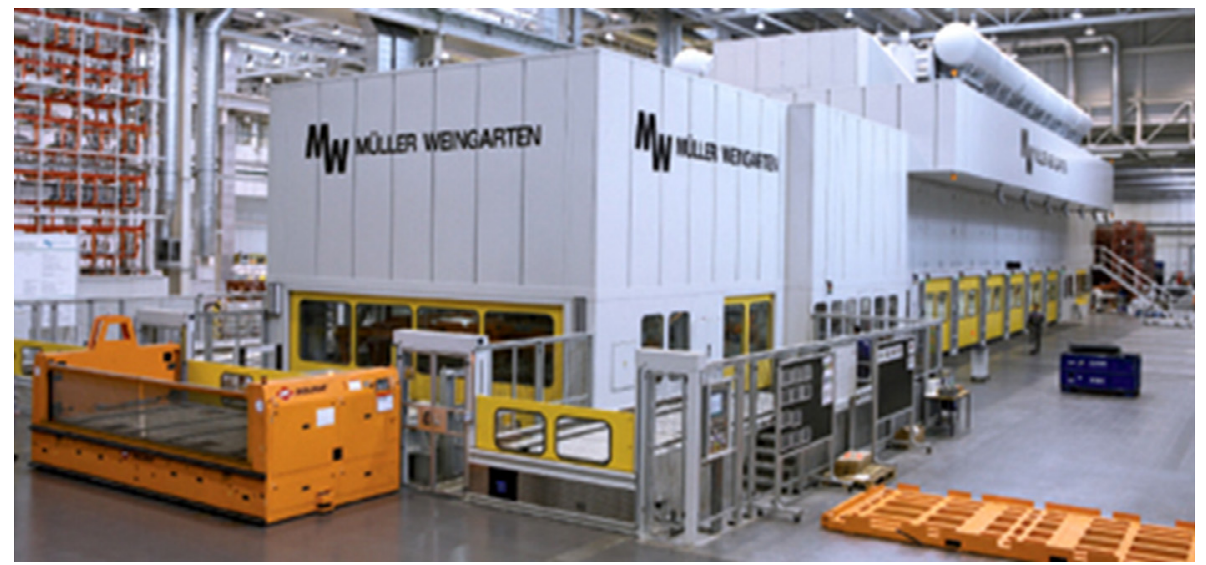

Figure 2. High volume "Compact" Automotive crossbar press (Schuler Automation) [17].

"Robotizing the man" [15]. A more fundamental approach which relates more to the state of the art is a system such as that promoted by Schuler Automation [16] involving a completely integrated transfer press line.

This system uses a fully enclosed approach and a central drive to co-ordinate manufacture (see Figure 2). The part handling is achieved internally by using a modular lever transfer arrangement in order to handle formed panels between each individual press station.

A variety of handling systems are available such as cross shuttles, robot loading with conveyors, and independently controllable feeders depending on the client automation needs.

A similar retrofit crossbar approach is possible with a conventional press situation that transfers parts directly between presses without releasing the part. This should result is more accurate and reliable picking since the part does not undergo any intermediate drop convey and pick stage. Here the robot is mounted on a rail system and itself travels between sites whilst the part is still held (see Figure 3).
Offline robotic programming is available via systems such as MasterCam [18] Roboguide [19], Robotmaster [20] or the open source ROS [21], in order to generate path control and servo instructions for the preferred robot system [22].

In robotic handling a key factor is the reliability of the system and integrators aim to show that their systems perform robustly. An example of a reliable system is the use of MTBF and some suppliers claim to have an MTBF of 60,000 h for robots at Jaguar/Land Rover, meaning that a particular robot would only be down once in every 15 years [24] (Figure 4).

Typical advertised benefits of robotic handling include:

improved consistency, greater production volume, reduced labour-hours, safer operation, lowered production costs, improved safety, environmentally friendly through lower energy costs, typical productive life of robot $10-15$ years [25]. 


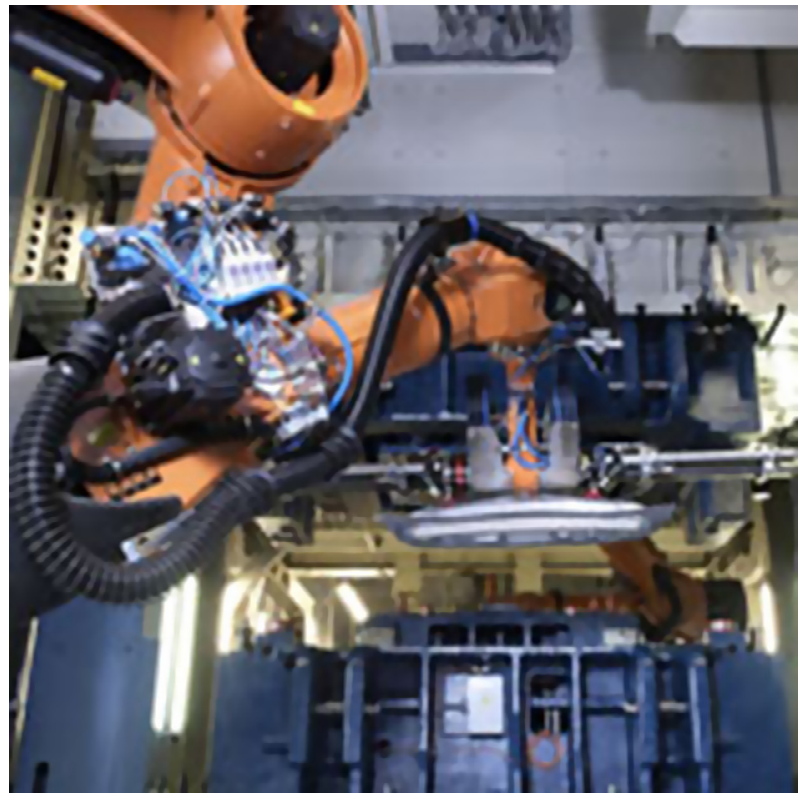

Figure 3. "Crossbar Robot" transfers parts directly from press to press (Schuler Automation) [23].

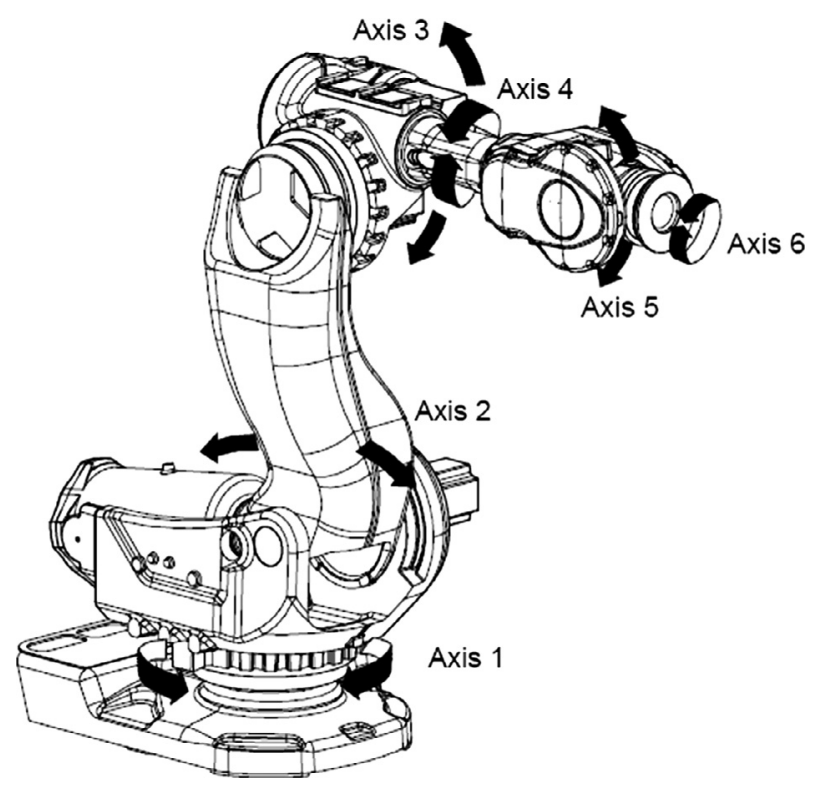

Figure 4. Standard 6 axis Robot model shown IRB 7600 (ABB).

Here the prevalence of high temperatures (up to $1100{ }^{\circ} \mathrm{C}$ for some materials) and airborne detritus means that exceptionally robust robots are used, often incorporating specially adapted grippers [26]. Note the use of caged robot enclosure for personnel protection (see Figure 5). Robot suppliers can provide systems with payloads ranging up to $500 \mathrm{~kg}$ or more depending on reach and are typically programmed via a teach pendant, though offline programming is often possible. A typical "6-axis" servo has changed little in the basic configuration over 2 decades [27] though have now become extremely reliable, though overall system integrity is more difficult to assure

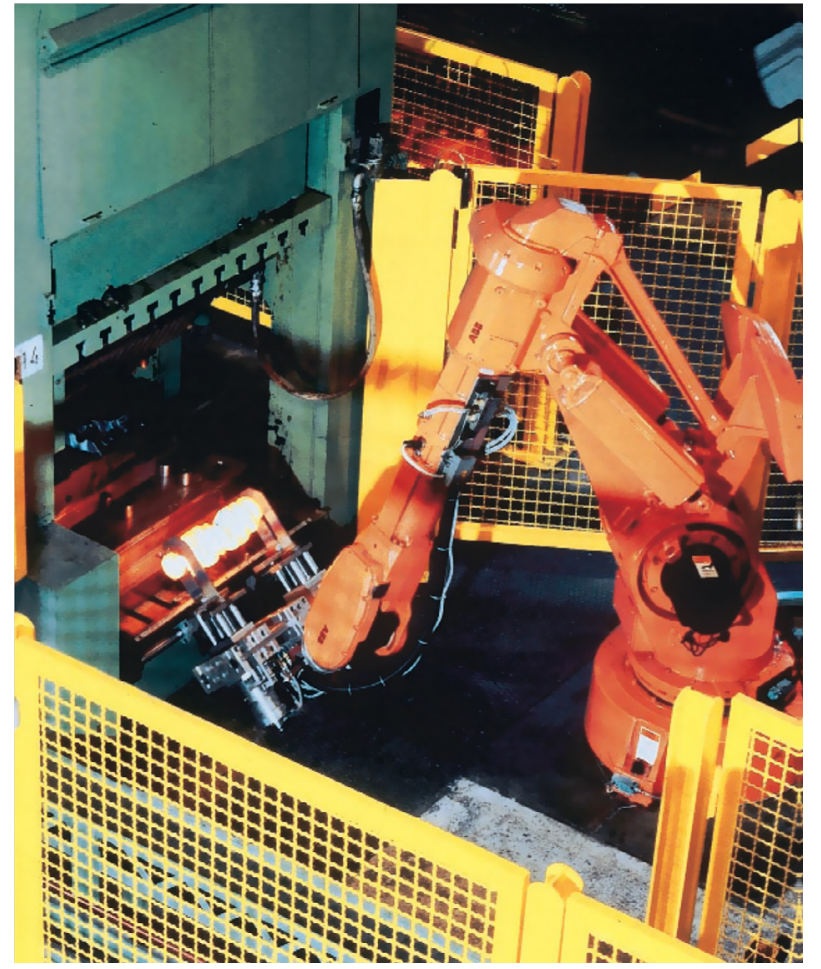

Figure 5. Robot handling crankshafts for the auto industry (ABB).

because of the many interactions that occur with real world devices in a forging cell, particularly during the setup cycle.

Note that the addition of vision guided packages, including 2D and 3D aspects are slowly becoming more prevalent though 3D vision is not suitable for all applications in forging and infra-red interference provides special difficulties as does lens contamination issues from free floating dag.

An excellent demonstration of a robotic forging cell for turbine blades was described in the "How it's made" series [28] which describes a robotic forge using a two gripper approach to manipulate slugs, and form performs which also integrates furnace heating, deburring and automated lubrication.

Note that Leistritz claim [29] that by using Superplastic forging in isothermal conditions (rather than super plastic forming) that a near net shape can be forged, used in conjunction with electrochemical-machining for metal removal. The stated Leistritz philosophy on automation relates to a common user interface and is really a user-centred control systems approach. This presents an alternative to substitutive thinking on automation which has the overall goal of minimising human interaction and is based largely on a machine dominated process. Example Leistritz blades are shown in Figure 6.

\subsection{Automated lubrication}

Discussions with Houghton Plc and interaction with leading manufacturers reveal that automatic lubrication systems are often a part of the forming process - usually named "dagging". This applies a colloidal graphite based suspension of water and mineral oil [30] and one approach to optimisation is to use a 


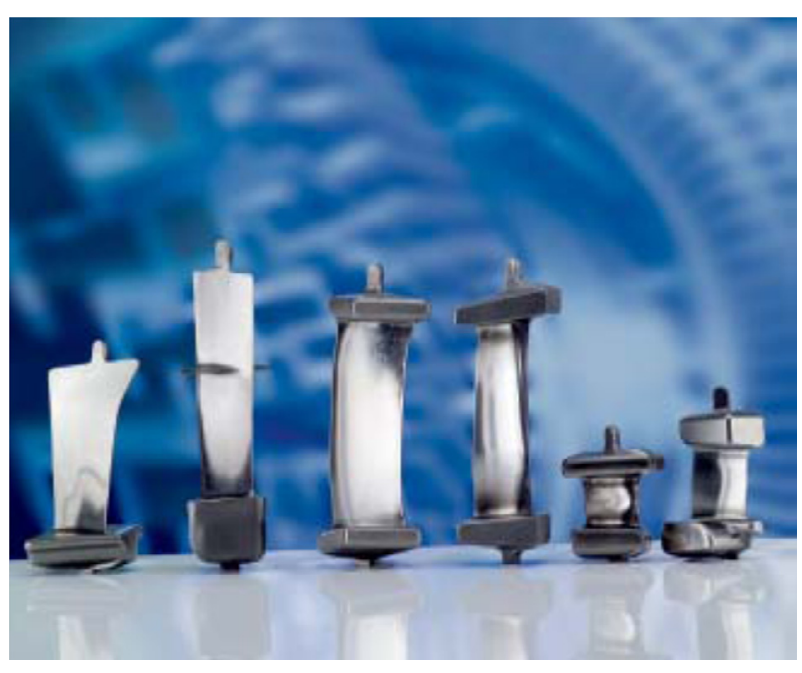

Figure 6. Forged compressor blades (Leistritz).

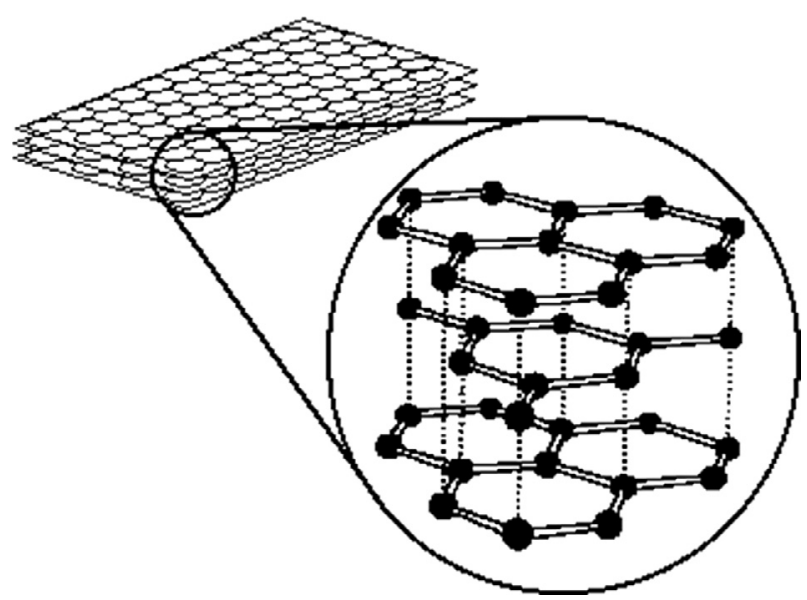

Figure 7. Crystalline graphite structure illustrating lubricious cleavage planes (McIntosh).

Taguchi or design of experiments approach. A diagram illustrating the structure for graphite dag is shown in Figure 7.

Once the parameters of the specific concentration have been determined for a particular parts configuration (e.g. extrusion and heading) and temperature then Statistical Process Control (SPC) can be used to manage the process. An example of dag application for extrusion is shown in Figure 8.

Many presses include auto-lubrication functions however these do not always carry an approved "CE Mark" [31] and for this reason manual dagging is relatively common. Obtaining a CE mark for the particular auto-lubrication press is a costly and time consuming process and one best undertaken by the press manufacturer at the time of the build, therefore manual dagging is likely to continue until an uncertified pre-existing press is replaced by one having the relevant certifications. There also a perception that when it comes to the application of dag that the operator knows best.

Surprisingly in automation applications the dagging function seems to be persistently applied by the human operator.
A series of videos on forging illustrate this and very often a manually applied spray process can be seen entering the field of view from the side. The illustrated cell in this video of truck axles forging shows robots moving on a floor mounted rail in a two stage forging process however the dag is still applied by an operator [32].

One novel application for a retrofit is described by East Engineering [33] similar to that of an inkjet printer (see Figure 9). Here the dag is directly programmed via a series of small nozzles to spray particularly on one area and developed by "Jerko Sprue-system [34]". The advantages being very fine atomisation, precise delivery, and cooling effects limited to only where the dag is applied meaning a more stable process (see Figure 10).

This system has been installed by DIC Engineering as part of a crankshaft line for General Motors incorporating an NKMZ $7000 \mathrm{~T}$ forging press, with piezoelectric load cells to protect against overload.

It should be possible using a robot spray technique to generate a relatively sophisticated dag spray pattern that only operates where it is absolutely needed using a modified lance rather than in the general area of the part and tooling. This will result in less dag being applied together with reduction in the associated cooling effects, and could be of use particularly for blade forming.

Some of the ideas presented in the above review were distilled into an example automated cell illustrating the key features, and is presented here as a case study, in the Advanced Forming Research Centre (AFRC) at the University of Strathclyde.

A key issue to be addressed in forge manufacturing is that of the variability introduced by manually controlled methods. Individual forge operators tend to use individualised technique which means that the output varies according to the speed of the operator, the amount of lubricant applied and leads to results which can be inconsistent. Automation of this process, as well as leading to increases in production volume additionally gives improvements quality and especially in consistency of output [35]. For the research investigator the latter feature (related to stabilisation of experimental conditions) - means that the automated process can then itself be used as a process debugging tool, without the confounding that tends to obscure the process improvements obtainable from any particular change. Therefore the automated system itself can be used to diagnose the process, for example where new consistent defects start to emerge from a stable process. Where manufacturing operations consist of both manual and automated processes improvements can be reflected back from the automated to the manual operations in terms of for example, the application of lubricant.

The introduction of Automation in forging clearly has a number of specific challenges. These are described in the following section.

\section{Research challenges in forging automation}

There are a series of important challenges that impact on industrial implementation of forging automation including 


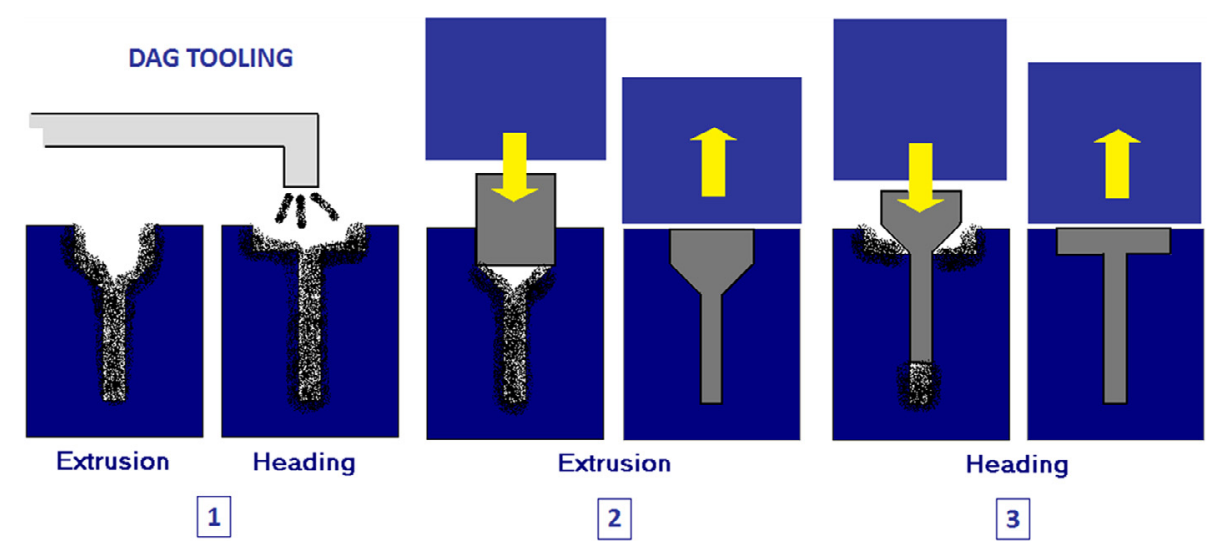

Figure 8. Dag application in extrusion and heading (McIntosh).

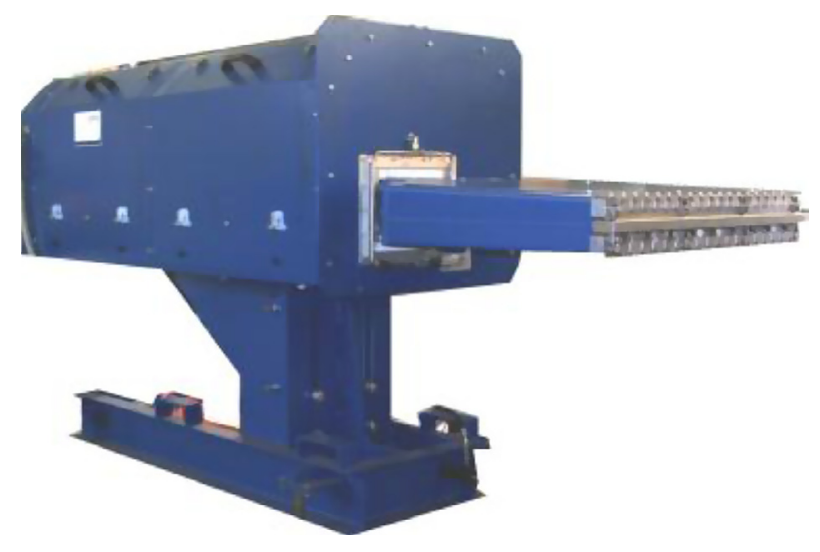

Figure 9. Jerko Dag “printer” (Jerko Spruesystems).

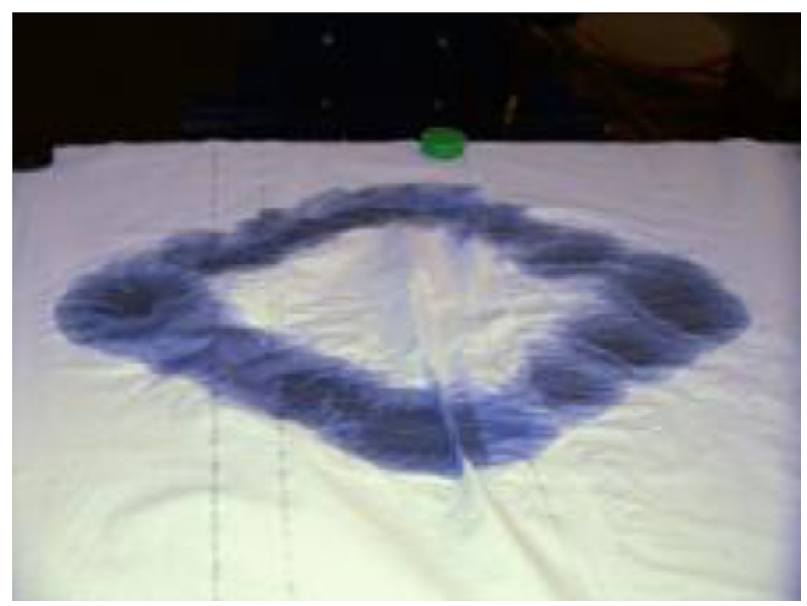

Figure 10. Printed Dag pattern showing local directed spray (Jerko Spruesystems).

potential financial benefits [36] such as return on investment, Payback period, which interact with specific technical ones, which are described here.

Overall these can be grouped into Process Specific Challenges, and No specific challenges applicable to any automation project.

\subsection{Non specific automation process challenges}

It is critical to any technical project that the basic groundwork required by a long term effort investment is laid by obtaining full backing from senior management. The issue is that if there is insufficient or lukewarm support at senior production levels then when unforeseen difficulties arise, and especially where there is no immediate solution, the danger is that the project will be stopped. It is also important to have an extremely complete understanding of the detailed nature of the task that requires automation in order to be able to specify and implement an automated system. Barriers to implementation can include fear of redundancy [37] and labour displacement - though in practice robotic automation tends to mean that existing staff are redeployed into other areas after an increase in volume and quality has been achieved, though some of those who leave voluntarily may not be replaced. There can also be a process knowledge gap on an operation, and a standard technique is to video the precise manufacturing operations as well as to elicit knowledge form the particular operator, some redesign of the task is often required in order to cope with the limitations of the automation system. This is against the known background that robots are not "accurate" in the sense that CNC machines are accurate, but the repeatability and flexibility means that it these limitations can be handled and still make a productive system.

Nevertheless we focus here on the technical aspects of implementation, assuming that the solid financial and particularly managerial backing exists. Automation in general has a range of challenges but forging presents some that rare relatively uncommon. There is a market led desire to move towards small smaller batch sizes [38] and the case for automation may mean that the technical aspects of implementation cannot easily be demonstrated - technical achievability influences the benefits.

The major long term strategic challenge involved is that of worker substitution, the original source of the word robot [39], particularly applicable in a hostile environment such as a forge or foundry operations involving high temperatures, levels of contamination, and extremely powerful machinery. The next great leap forward is the deployment of the Asimo style selforganising robot-android that has a capability without being 
micro-managed at every step of the production process, and ideally capable of easy redeployment between processes. Even basic self organising systems indicate that that this is some way off in the future [40], nevertheless the goal is to provide plug compatible replacement, at lowered cost and raised quality. Therefore the benefits of automation can be gained without the inherent disadvantages of bolted down arms with smart sensors, programming and end effectors, slaved to a master controllers which comprise the bulk of automated systems today. Such systems are at least a decade away or more from being deployed in manufacturing.

\subsubsection{Teamwork/collaborative approaches}

A challenge for the research practitioner is that of robothuman interaction at a number of levels. The majority of robot programming is still done in practice via the traditional teach pendant - Illustration - backed by vendor training courses, heavy course documentation and support channels. The reason for pendant based operation is that the real world is still difficult to model at the sufficiently detailed level in order to allow true offline programming to occur. This means that advanced. Program capability is thus dependant on high levels of familiarity with the various menu trees, detailed co-ordinated systems, Tool coordinate variants, in variable declarations, screen prompts and the like. In short it is still fundamentally a programming task requiring programmer skills, which present challenges for the occasional user/and often it means that external expertise has to be bought in to complete a specific task in a scheduled time, especially when production downtime is involved.

Recent alternative approaches to aspects of robot programming include development by KUKA and others of on passive click and drag and interaction via Mobile phone devices. One approach is simply to emulate the pendant software [41] inside the application, though other emerging methods include physically moving the robot itself [42] into position and selecting a point recall button on the robot [43]. This offers a much more direct way of programming the robot since humans can use intuitive honed skills in our own natural way of controlling motion rather than via an artificial menu system - reducing programming and development time. Here falling hardware costs mean that entry level industrial robots, which start at about $£ 20 \mathrm{k}$, may be only $20 \%$ of the cost of an installation due to the additional value-added services that are required to make an installation actually function correctly. That is the area that key research challenge to simplify the introduction of smart flexible robots.

Accuracy - robots are not "accurate" - or as accurate as a typical CNC machine tool, however they are conventionally classed as "repeatable" which allows advanced systems to be successful.

\subsubsection{Human robot collaboration/collision}

It is expected that next generation robotics for manufacturing is going to interact more directly with the human operator and advanced safety protocols and ways of working are currently being developed. The idea is to move back from the caged robot process to one where human and robot work cooperatively and using the humans sophisticated sensory and knowledge capability rather than automated substitution. This means that intrinsic safety protocols such as advanced sensing in order to manage torque and speed control are required if the human is going "back in the cage". One approach is to have a set of whisker detectors so that if a human (or other unexpected object) is encountered the robot immediately reduces power so that the human is not hurt. One system currently operating (without a set of guarding) is described by Kuka where the human works closely with the robot - augmenting the robots ability to perform repetitive tasks with the human ability to sense the world, make decisions and to behave in a nonprogrammed way. The trend is towards more bottom up rather than top down robot implementation [44] see ISO Tech Specification TS 15066 [45] with robot safety via collaborative interworking [43].

For part or product family opportunities where the investment case is reasonably demonstrated difficulties include the following items.

\subsection{Process specific challenges}

Hot Forging in particular means that handling equipment must be designed to cope with metallic materials at up to $1100{ }^{\circ} \mathrm{C}$, so replaceable, robust handling systems such as gripper jaws are one possible solution for long term use. Cold and warm forging often means a commensurate increase in forging forces. The idea that fragile dexterous robot hands can be used is not one that finds implementation in practice. Hence smart techniques for advanced handling or sensing must be sufficiently robust as to cope with locally high temperatures. In the case of picking from for example an Electrotherm [46] rotary furnace, the entire structure (end effector/gripper/part) is soaked for up to a second in an oven temperature typically greater than $1100{ }^{\circ} \mathrm{C}$. The level of robustness for any equipment to deal with these temperatures should not be underestimated, and in super-plasticity high temperatures are encountered in combination with saggy, tricky to handle and position materials, often in combination with specific cleaning requirements due to uneven deposition of slag. Additionally the volume rates involved are often relatively low, and require a level of skilled haptic feedback that is not commonly found in industrial automation, though research is moving forward rapidly there. Again the issue relates critically back to the business case for the investment in a manufacturing context. Note that whilst manufacturing accounts for about $10 \%$ of employees it accounts for $18 \%$ of fatalities, and $18 \%$ of seven day injuries [47].

Extremely high pressure/forces - upwards of 100 tonne Force are not unusual. Whilst this means that there is an opportunity to improve operator safety particularly with regards to presses - a tangible benefit - it also means that extremely close attention has to be paid to forge interlocks between the robot and the press. Note that where press injuries occur a large 
percentage lead to hand amputations [48]. Even smaller presses are easily capable of crushing a handling or lubricating robot which means that the system has to have extremely reliable methods of controlling the operations in the tooling area. A previous research project $[49,50]$ where a cold forming press routinely operated at 300 strokes/min (and on occasion up to 1000/ min) described a programmable method of co-ordinating the motorised feeding cycle with the instantaneous linear encoder position on the press, though commonly in addition limit switches are used which interact with the master cell PLC to co-ordinate the robot. This means that for example simply relying on visual indications given the difficult operating environment, which often consists of poorly lit, and variable ambient conditions, presents high risks to equipment and personnel.

An additional challenge related to automatic forging/forming is that loosely contained objects have the potential to be ejected at very high speed from tooling. One of the major causes of injury related to presses [51] is the secondary damage caused by potential high temperature hard projectiles being ejected from the press. This can occasionally occur in the use of ceramic tooling for superplastic forming (SPF) presses temps $450{ }^{\circ} \mathrm{C}-950{ }^{\circ} \mathrm{C}$ at up to $7 \mathrm{MPa}$ forming pressures for Ti-6AL$4 \mathrm{~V}$, though redesign techniques have mitigated the tendency of the tooling to explode. The Boeing Company, [52] reports two occurrences of catastrophic explosive brittle failure in 14 years where serious injury was prevented by reinforced heat shields.

Airborne lubricant - often forming and particularly forging tends to use lubricating/release agents and the result is that equipment is covered in a thin dusty black film of graphite. This detritus also presents difficulties for the picking and placing operations since the compacted/layered material tends to strongly adhere (at high temperatures) to the tooling. In manual situations this is dealt with by intermittent cleaning based on operator knowledge of where and how the build-up occurs, however in an automatic context provision has to be made otherwise toolsets become damaged as the effective cavity volume reduces [3].

Handling/haptic dexterity - this can be a particular research challenge where dexterity and adaptation is required and the handling and automation of SPF is an area where automation has been proven to be difficult [52]. Dealing with non-rigid formed structures (e.g. SPF) presents handling challenges that low sensitivity robots often have difficulty with, allied often to lower volumes of very high value parts - this is often linked to a tricky cleaning cycle due to deposits being left on the tooling. The operator skill level is relatively high and the process is not always completely understood which presents difficulties for the automation engineer, or research challenges. Current handling approaches are most frequently based on the use of high temperature protective "silver" suits whilst the operator stands in front of a $1000{ }^{\circ} \mathrm{C}$ oven and uses previous skill and a delicate sense of feel to remove the super-plastically formed sheet with basic long tools.

One potential approach is for active force measurement, or current feedback/monitoring to be used allied to an advanced vision system. Here again the difficulty is based on the high temperatures encountered.

\subsubsection{Handling flash/excess material}

Forging and forming processes often produce small but important amounts of waste or excess flash material either after clipping or as part of the extrusion process. One objective of the forming process is to optimise the material parameters by using the deformation process and thus the idea is to do the minimum possible machining. If clipping operations are used the build-up of flash can become a serious issue as it the one of the parts simply remain stuck in the tooling so that the handling system cannot remove them. Some detailed and practical thought needs to be put in to work out how the material will be dealt with, either via ejector pins or similar approaches.

\subsubsection{Press integration}

Integrating with existing manufacturers presses presents major challenges and one approach is to go back to the original manufacturer, or specifically the press controller. Presses have long lives typically 50 years + but much longer is not unusual - with upgrades in controllers in order to interface with advanced metrology, (displacement, force measurement typically) often via industrial style PLC interfaces. Fortunately specialists exist that can deal with this, skills which are often employed by the systems integrator, including the critical safety elements, safety interlocks, guarding as required [19, 53, 54]. Often once the press project moves forwards snags occur, such as dealing with the waste product (flash) created when parts are reduced in volume - failure to do this will jam the tools, as well as how parts that stick in the tool are to be removed. One method of anticipating problems is to generate a detailed video of exactly (in excruciating detail), what goes on in the typical formation of a part. Experience suggests that even if this is done the depth of the micromanagement of every single element of the process has to be focussed on in extreme detail. One hypothesis is: what systems have to be in place to allow a blind person do this task? Learning gained from that pre-analysis phase - will pay off at the implementation stage however some developments may only emerge later, here the use of a knowledgeable systems integrator is invaluable. Automated tool changing is also possible but tends to be extremely expensive, up to $50 \%$ of the cost of a new press in some cases, depending on mass, and here conventional Single Minute Exchange of Die techniques are more likely to be cost effective.

\section{Case study - advanced forming centre automation}

A forging automation research programme at University of Strathclyde has been developed in conjunction with input from world leading companies such as Boeing, Rolls Royce, Timet, Aubert-Duvall and Barnes Aerospace, and formerly, Mettis Aerospace. The industrial input formed a key part of the research agenda based on a membership model [55]. As part of that process a requirement to pursue advanced automation techniques was identified as part of a strategic road-mapping 


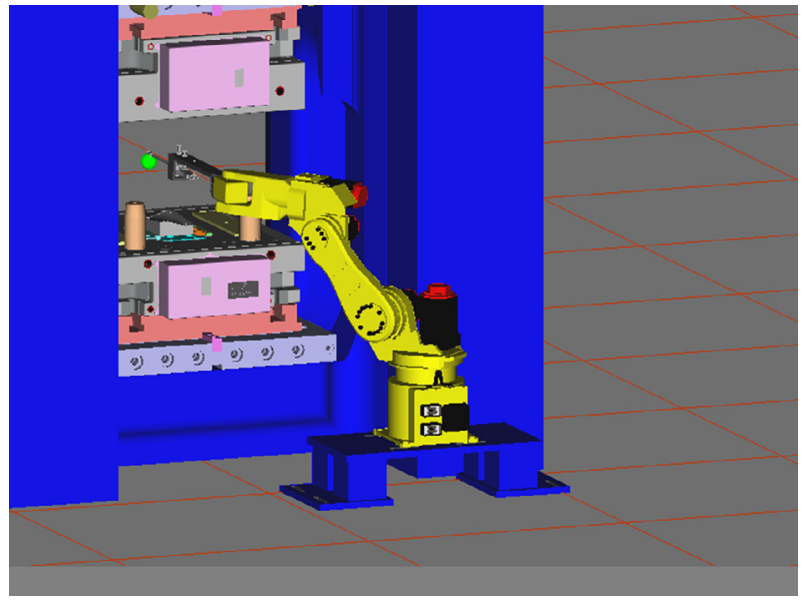

Figure 11. Manual spraying becomes robotically consistent.

exercise and this resulted in a creation by the author of a vision for Automation by the author to encapsulate the main objectives for Automation in AFRC.

It was clear from industrial interaction that manual variability in forging in particular is a key determinant of inconsistency in product quality and for a research organisation attempts to generate process improvements can be masked by the associated indeterminism. Some operators take longer to transport part from furnace to press, even a single operator has variability in the associated processing time dependent on levels of tiredness, and other external factors. Thus part temperature - a key determinant of forging quality and grain size is difficult to control at the point of hit, regardless of how much effort is placed on temperature control at the oven, since the transport time is variable.

Process variability in manually dominated processes has been widely observed by work study exercises and is a feature of simulation exercises in manufacturing $[56,57]$. For the process experimenter this variability is anathema in the isolation of improvements so as part of the technological development of advanced forging and forming. Accordingly the key vision of the AFRC automation then evolved into a two-pronged approach, that of developing and researching advanced automation techniques in their own right, and to attempt to expunge, or at least to mitigate, the inherent variability in forging techniques by repeatable, automated, usually robotic approaches.

This vision was then discussed and presented internally so that agreement on the strategic thinking could be embedded in the senior management of the industrial partners, key to assuring the success of the project. Management buy-in is essential from the earliest possible stage in order to ensure the viability of the project when the going gets difficult. A pilot project was selected see Figure 11, and detail associated with the project is presented in the following section.

\section{Vision for automation}

The author defined the purpose of Automation as:

1. To generate a stable repeatable platform for forging and forming trials/experiments.
2. To generate and prove out automation forming techniques which have a 5-10 year technical benefits lead on existing industrial practice.

These industrial and research benefits include the following key features:

increased production volume,

reduced variability,

improved quality - dimensional accuracy,

Reduced Cost Reduction in scrap,

improved safety "Power presses are among the most dangerous machines used in industry" (PUWER) [58], process debugging capability via stabilised experimentation.

\subsection{Design considerations}

As part of the implementation of the Automation Capability a Forge working group was set up comprising forge operators, manufacturing engineers, in order to clarify the key integration strategies as well as the tactical implication of the various technological choices and how they would impact on the project. From this interaction it became clear that here was a desire to operate the main forge - a 2000 tonne max screw press from Schuler (Figure 16) in both manual and automated modes in order to perform relatively short production run research projects on special metals as well as to have an integrated automation cell. This meant that from a design point of view the automation had to be capable of being safely stowed in the short term in order that particular manually orientated projects could be executed. For the Forging automation team his meant a fairly hard think about exactly how to balance the apparently competing requirements of both manual and automated operation.

For example in a typical robotic orientated cell a pick and place robot would be positioned front and centre (or slightly offset due to the geometry of the robot joints) in a permanent floor position, meaning difficulties for both access and interoperability. A possible configuration would be to have the handling robot on a swing arm or similar system to allow it to be deployed when necessary, however this has implications in terms of trailing cables and repositioning and after some consideration an overhead gantry was selected which allowed the handling robot to be safely parked when manual only operation is desired.

As part of the investigative work it was decided to pilot a particular project in the forge handling area and a Tier1 membership poll was taken in order to confirm that this was an acceptable approach, duly ratified by the AFRC technical board. The pilot project selected was automated lubrication \& cleaning colloquially known as "dagging" based on the application of a specialised micro-graphite suspension to the tooling. Here consistency of application and lubricant amount on to the tooling was a key objective as well as to develop some learning in the forge on the practicalities of working with industrial scale applications.

The first step was to define what the characteristics of the automated solution was to do which included the area of the 
tooling to be covered and the reach of the robot - bearing in mind that the recommendation is to reduce exposure of the robot itself to the operation of the 2000 tonne press in case of inadvertent crushing. An automated stirring system was also introduced (agitation) in order to prevent the suspended graphite in the lubricant from settling out into layers. In manual operation an occasional quick stir with a stick does the job but all these small steps have to be automated in an integrated installation.

The first step was to trial various locations based on particular lubricant spray gun choices, and based on this possible selection in conjunction with a possible adaptor to cover the entire area, a design and manufacture of the associated holding fixtures/nozzle assembly.

Once a first estimate of the robot assembly had been created it was then modelled in the Fanuc Software "Roboguide" [59] which contains a detailed model of the each of the Fanuc robots, and can generate motion paths and timing information for the particular type of robot chosen. It is also particularly useful as a communications tool since it can show exactly how a particular robot will work in practice to each member of the team and create supplementary AVI video material (see Figures 11 and 12). Feedback on the simulation was then incorporated into the model and a suitable mounting for the robot was then defined based on the technicalities of positioning a plinth onto a robot pit - defined prior to the automation theme. A student was engaged for this work and having completed the modelling work in ProEngineer can then be exchanged into Roboguide, an approach used in other Fanuc installations [60, 61].

A key part of the design of the manufacturing process was to break the process into a series of automatable tasks which could then be controlled either by the robot controller through conventional $24 \mathrm{~V} \mathrm{DC} \mathrm{I/O} \mathrm{or} \mathrm{alternatively} \mathrm{via} \mathrm{a} \mathrm{master} \mathrm{PLC,} \mathrm{to}$ be installed at a later date. In the first instance the development has been focused on controlling I/O via the robot controller since that was convenient and available, though these can migrate to the master PLC later.

Breaking the task down into individual parts means that it was realised that what the forge operator does is more involved than realised and includes a suit of operations so that the following devices have to be slaved to a master control program.

\subsubsection{Furnace heating - automation}

The AFRC rotary furnace device includes an incrementing hearth which can be moved round to the next position for presentation to the operator/robot.

\subsection{Screw press - automation}

Here the screw press needs to be controlled remotely by a master PLC in order to execute pre-programmed load conditions and press stroke. Some thought needed to be put into what would happen if a part is stuck in the tooling, as well as into making sure that appropriate communications protocols are put into place and that facilities exist to interact with the master PLC. In the case of the AFRC press it was part of the tender process that the press was automation ready, but often some

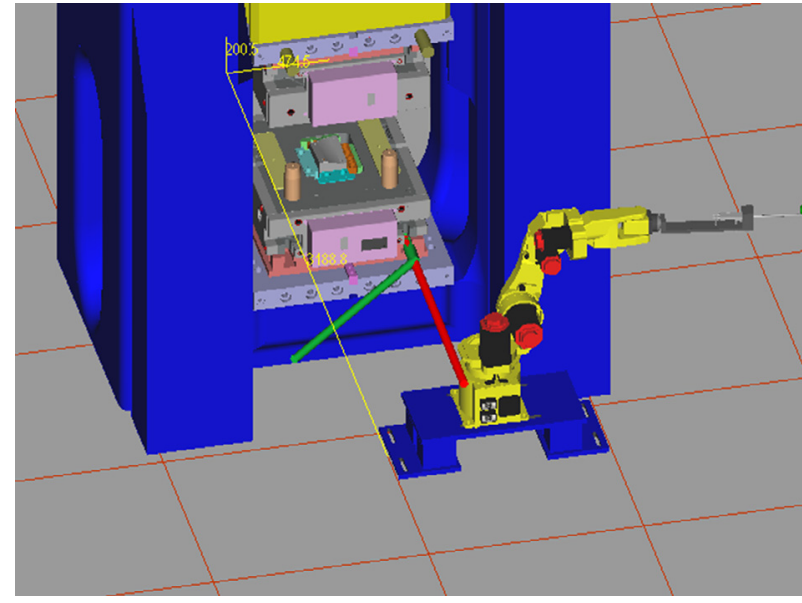

Figure 12. Design of special tool - layout checking.

kind of control retrofit is required on major items of capital equipment like this. Note that large presses in particular can have a useful life commonly 50 years or more, and controls upgrades are a regular feature.

\subsubsection{Presentation devices}

Part of the issues of using industrial automation is the level of investment that is required in presentation devices and this aspect needs careful appraisal - and an acceptance that fine tuning will probably be required after the system has been introduced. Since the handling and orientation devices have to strictly aligned on the basis of a pick repeat operation (often without a vision system) then either some sort of vibratory bowl feed system is used or other arrangement to present parts in a consistent location.

In the implementation here since the setup is not for continuous industrial production in the first instance it was decided to manually preload the furnace with billets and then drive the automation from that point. This offers flexibility and does not detract from the benefits of automation since the process only really begins after the billets or preforms have been heated to the correct temperature.

\subsubsection{Lubrication robot Fanuc M6iB/6S - basic specifications}

$951 \mathrm{~mm}$ reach,

$6 \mathrm{Kg}$ payload at wrist, upright, Wall or Inverted mounting, $0.08 \mathrm{~mm}$ repeatability at full speed, 8 Digital Inputs/Outputs, mechanical weight $135 \mathrm{~kg}$.

An examination of the setup of the press lubrication at the strategic level allocated the dagging robot and a convenient robot supplied by Fanuc was available the M6iB/6S. Since it was recommended that the robot itself not be placed at any time within the jaws of the press, it was sensible to use one with a relatively long reach since the platens of the AFRC screw press are large and it was desired that the robot be able to lubricate as 


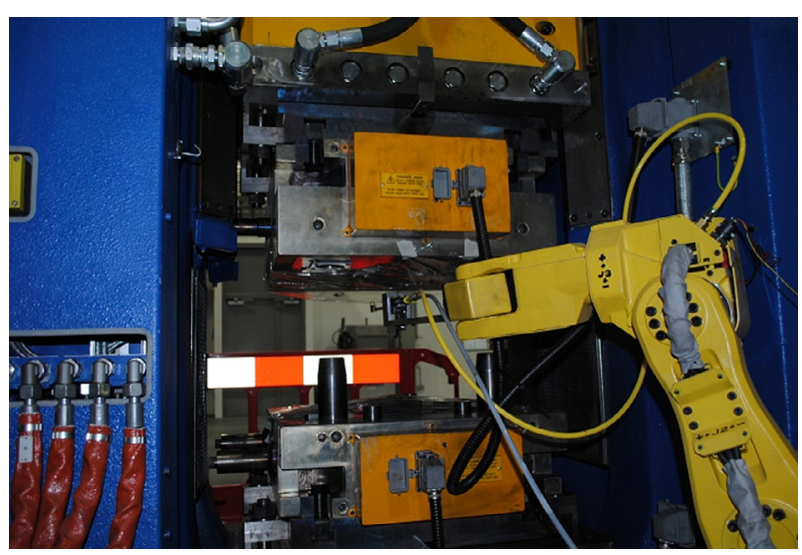

Figure 13. Robot operating inside press tooling.

much of this as possible. The other robot that was initially considered an LR Mate used elsewhere [3] would also have been feasible for smaller forgings but would have provided less coverage of the complete area of the tooling. The M6iB also provides fairly sophisticated Robot $\mathrm{I} / \mathrm{O}$ that can slave associated devices without the need for a PLC - useful at the prototype/ pilot stage in particular. A photo of the setup is shown in Figure 13.

It was necessary to analyse how this would work with the particular choices of lubricant gun available and how that would interact with the screw press and associated obstacles - this is where working between the Software Roboguide/ ProEngineer proved invaluable, with $\mathrm{CAD}$ data being supplied by subcontractors in order to check the reach and manoeuvrability of the robot, and the required press opening to allow it.

Part of the work that the manual operator does in forging is the application of lubricant, and one popular trade-name is "Dag", a range of technical fluids specially formulated for forming and forging operations. In practical terms the operator usually uses judgement to determine where and how much dag should be applied but a simplified path using the robot - akin to a spraying application is possible in order to obtain complete coverage. It is possible to tune the process - often using compressed air to drive a purpose made spray gun as well as a mixture screw which defines the size of the opening orifice and hence flow rate. Additional debugging of the re-spray pattern is available by close and knowledgeable examination of the parts produced to see if recognisable faults are apparent through the misapplication or over/under-application of lubricant. Here it is important that an experienced operator provided feedback on exactly how specific faults are being produced and what they mean.

In the case of the work being reported here the lubrication robot provided a convenient pilot project since it incorporated the main features of robotic operation without the precision required of a classical pick and place operation, and all the associated feeding supply devices.

\subsubsection{Safety protocols/guarding}

Legislative requirements and basic consideration for staff safety dictated at an early stage that safety guarding had to be included. Since even the smaller robot (overall mass $140 \mathrm{~kg}$ ) is capable of moving at up to $600^{\circ}$ per second it does have the potential to cause some damage both to staff and itself and auxiliary equipment. Guards were therefore procures and installed internally - having physical interlocks in the first instance, with plans to integrate electrical integration once the final PLC was selected. Final layout is contingent upon placement of the multiforge/horizontal upsetter and integration of the guarding to cope with both the position of that in the one manufacturing cell.

\subsubsection{Automation in practice}

As the development of the automation work continued it became obvious that the experimental benefits from highly repeatable trials of particular forging situations excited research interest. An additional project of opportunity arose, related to quenching where the advantage of repeatedly quenching an object under programmable control emerged. Despite the fact that the automated handling system was not designed for this from the outset a brief model in Roboguide and clarified that it would be possible to repeatedly quench objects, thus improving the consistency of the experimental procedures. This has been a trend that has been observed elsewhere, robots tend to be redeployed to other factory/R\&D functions rather than be replaced.

As part of this work it was decided to add automation capability to a larger furnace presently used for quenching trails based on a forklift. This means that it would be possible to routinely and repeated quench the relatively large (up to $25 \mathrm{~kg}$ ) objects in a systematic way, since the time to go from the furnace to quenchant by the existing manual (forklift) method was very variable.

\subsubsection{Trimming/clipping press}

At the output side of the screw press, a clipping press has been placed which removes any final waste from the final stamped part. From the Automation engineers view both presses can be regarded as similar devices - in which program control is handed over to the individual press controller, which then has load displacement parameters required for a particular part sequence.

\subsubsection{Robot gantry}

It became apparent from the design and review meetings that it would not be possible to have the pick robot at the back of the press for a number of reasons and this posed the issue of access:

the press was fixed in a substantial pit and could not be moved,

Fanuc M710iC,

$50 \mathrm{~kg}$ Max load capacity at wrist,

repeatability $\pm 0.07 \mathrm{~mm}$,

reach $2050 \mathrm{~mm}$,

mechanical weight $560 \mathrm{~kg}$,

6 axis electric servo robot,

capable of floor wall or ceiling mount capability. 


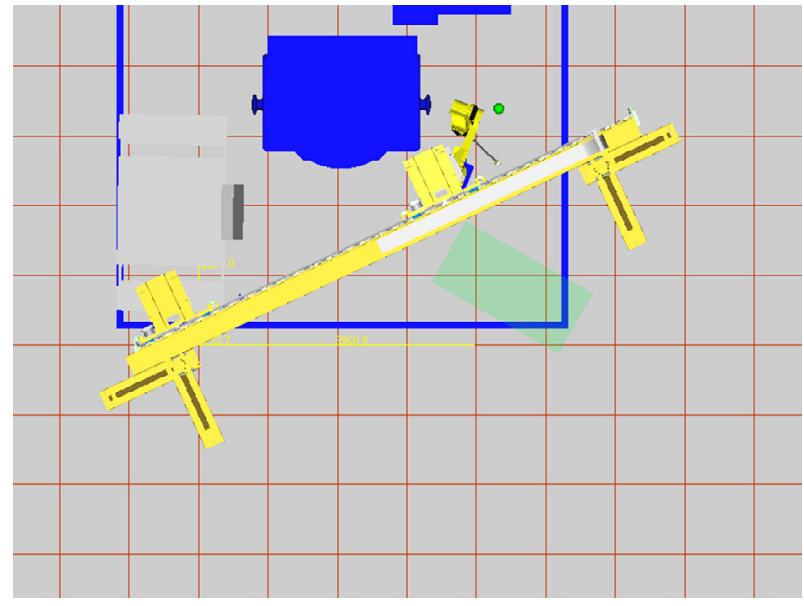

Figure 14. Checking clearance of robot and park position.

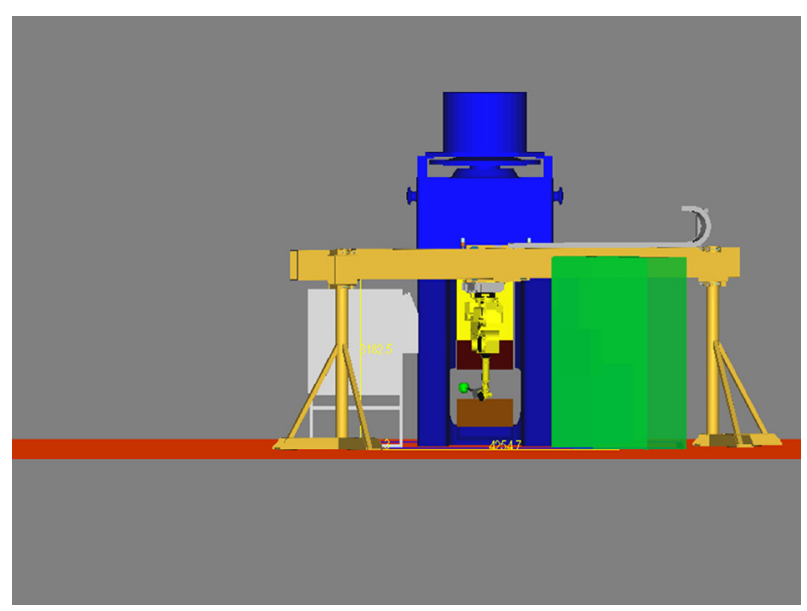

Figure 15. Checking robot interaction with press.

The Electrotherm Furnace was required at the front for manual operations - therefore the robot had to accommodate this too. Therefore the robot had to go at the front. The forklift could not access the rear of the press to change tooling - since that was the case the dagging robot was placed at the rear offset as shown.

The forklift based bolster/tool change mean that any temporary placement of the robot had to also cope with tool-change and no overhead crane was available. This meant that access to the front and the floor area had to be kept clear for both manual operation and for forklift access/egress. Placing the Robot on a swing arm was also considered though this would have meant considerable space implications as well as placing the $500 \mathrm{~kg}$ robot mass on the end of a simply supported cantilevered beam. Fanuc Roboguide was used to define the layout (see Figures 14 and 15).

Possibilities of mounting the robot on temporary plinths, on a fixed overhead structure were considered but a useful approach was that of inverting the robot and mounting it on a motorised gantry. This has a number of advantages.

The standard workspace is clear and the robot could be parked safely well away from the press when not in use.

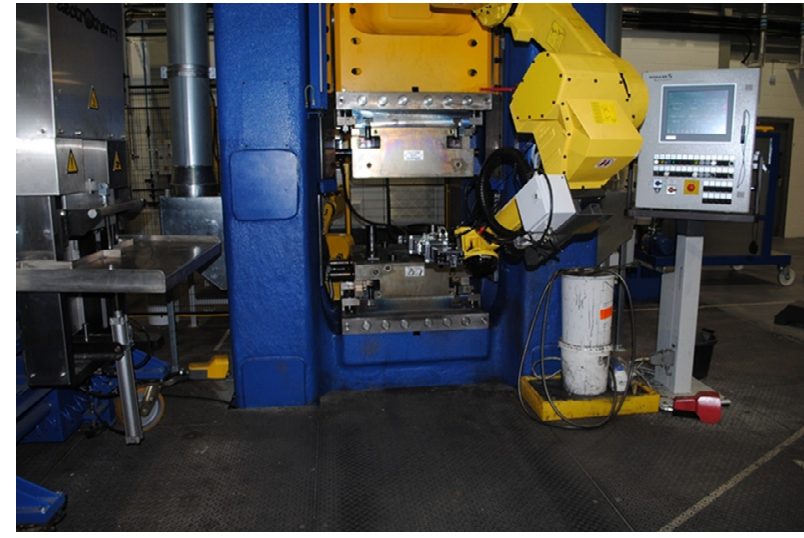

Figure 16. Fanuc M710 robot mounted inverted on Gantry.

The robot could be repeatedly placed in a centralised position when required. The working envelope of the robot and its flexibility would be enhanced considerably.

The disadvantages were the increased cost and increased complexity in programming ( 7 th axis).

However these disadvantages were greatly enhanced by the increased flexibility - especially important in an R\&D environment where the ability to change over from task to another and to implement small batch sizes is critically important. Having considered these alternatives the decision was taken to source a motorised overhead rail/Gantry via Fanuc robotics and the installation of this was also managed by Fanuc robotics, with simulation done in Roboguide and laid out using ProEngineer as shown in Figures 15 and 16 and basic configuration in Figure 16. This allowed the location and handling arrangement to be checked so that the robot could be placed in an ideal park position, and deployed in front of both the Programmable furnace, screw press and clipping press. Motion between these was able to be checked and elementary off line programming ideas looked at top check that the robot would be able to pick parts and place them correctly.

\section{Conclusions}

This paper has described a review of manufacturing automation as related to forging and an outline of a process to design and implement an automated robotic forging cell, based on a large press. The process requires clear management support from the beginning as well as a realisation that for certain applications smart design can accommodate both manual forging and automated forging operation using the same installation. A micro-management approach to the process is required that includes all the corrective actions that intelligent operators do in order to produce smart forgings and the process of laying out the cell has been described, using CAD tools including Roboguide, and ProEngineer. At the time of writing the cell is still undergoing development but the fundamentals have been laid down based on the original authors' vision and are expected to produce stable experimental results as well as a basic structure for flexible technology development. 
Acknowledgements. The layout and conception of this cell was assisted greatly by the following staff: $\mathrm{Mr}$ I. McLarnon, Mr J. McBain with additional contributions by Mr S. Cunniffe and Dr. M. Rosochowska.

\section{References}

1. C.S. Harrison, I.G. Ross, M. Bell-Jack, P.W. McEwan, Manual on manufacturing systems simulation for NCR Dundee, University of Dundee, 1989, p. 180 (unpublished).

2. Industrially orientated software to address cost benefits, http:// www.vanguardsw.com/products/vanguard-system/cost-modeling. htm

3. Automated forging of Gas turbine Compressor Blades from billet to finished forging, http://www.fanucrobotics.co.uk/en/ countries/fruk/news/rolls\%20royce

4. G. Chen, H. Wang, K. Zhao, Z. Lin, Modular Calculation of the Jacobian Matrix and Its application to the performance analyses of a Forging robot, Advanced Robotics 23 (2009) 1271-1279.

5. C. Yan, F. Gao, W. Guo, Co-ordinated kinematic modelling for motion planning of heavy duty manipulators in an integrated open die forging centre, Proceedings IMechE Part B: Journal of Engineering Manufacture 223 (2009) 1299-1313.

6. K.W. Lilly, A.S. Melligeri, Dynamic simulation and neural network compliance control of an intelligent forging center, Journal of Intelligent and Robotic Systems 17 (1996) 81-99.

7. X. Wang, M. Yukawa, Y. Yoshita, T. Sukeda, T. Ishikawa, Research on some basic deformations in free forging with robot and servo-press, Journal of Materials Processing Technology 209 (2009) 3030-3038.

8. R. Badadou, H. Shimahara, G. Hirt, Automated semisolid forging of steel components by means of thixojoining, Solid State Phenomena 116-117 (2006) 383-386.

9. http://www.forging.org/pdf/TechPlan2006-2007Rev.2.pdf

10. http://www.ogtechnologies.com/hoteye.html

11. http://www.bara.org.uk/

12. http://www.bara.org.uk/info/statistics/Industrial_Robot_Facts 2010.pdf

13. Jan Hutson, Forge Shop Automation, part 1, April 2009, pp. 20-23, www.forgemag.com

14. Jan Hutson, Forge Shop Automation, part 2, July 2009, pp. 23-26, www.forgemag.com

15. Robot - from Rossums Universal Robots - Capek, translates as "hard worker" or even "serf labour".

16. http://www.schulergroup.com/major/documents/downloads/ download technologien branchen produkte/download technology forging/massivumformung_broschuere_e.pdf

17. http://www.schulergroup.com/major/us/11_Highlights/20_Compact Crossbar_Pressen/index.html

18. http://www.mastercam.com/

19. http://www.fanucrobotics.co.uk/en/products/software

20. http://www.robotmaster.com/

21. http://www.ros.org/

22. Private conversation, G. Dr G. Pierce Centre for Ultrasonic Engineering, Univ. Strathclyde.

23. http://www.schulergroup.com/major/us/04_Presse/010 Pressemitteilung/2009/2009_02_05_crossbar_roboter/index.html

24. ABB Robotics "Foundry Magazine”, November 2008.
25. Retraining Robots, Ben Hargreaves, professional Engineering, 21st April, 2010, pp. 29.

26. http://www.abb.com/

27. http://www.rimrockcorp.com/files/News/ForgeShop_pt_2.pdf

28. http://www.youtube.com/watch?v=WIMzH87RWJM. Channel Series 9 Episode 3 segment D, Jet Compressor blades Episode 107.

29. http://www.leistritz.com/extrusion/en/04_products/ steuerungstechnik_2.html

30. L. McIntosh, Internal Rolls-Royce report, Department of Mechanical Engineering, University of Strathclyde, 2009 (unpublished).

31. http://en.wikipedia.org/wiki/CE_mark

32. http://www.youtube.com/watch? $\mathrm{v}=\mathrm{xHH} 24$ LCPR8\&feature $=$ related

33. http://www.east-engineering.eu/Segment\%20Spray\%20eng.pdf

34. http://www.jerko-spruehsysteme.com/index.htm

35. http://forgingmagazine.com/feature/2x-robots-doubles-productivity

36. P.S. Adler, Managing Flexible Automation, California Management Review Spring 30, 3 (1988) 34-57.

37. Professional Engineering, Institution of Mechanical Engineers 27, 5 (2014) 27-28.

38. http://www.ibef.org/download/corporatebharatforge_june06.pdf

39. K. Capek, Rosumovi Univerzální Roboti, Science Fiction Play (1920).

40. N. Ay, N. Bertschinger, R. Der, F. Guttler, E. Olbrich, Predictive information and explorative behaviour of autonomous robots, European Physical Journal B 63 (2008) 329-339.

41. https://www.youtube.com/watch?v=yFi7UL70zTo

42. T. Hulin, C. Preushe, E. Yechiam, A. Telpaz, V. Schmirgel, U.E. Zimmerman, Haptic and Visual Training of system behavior a case study for Robotic Programming-by-Demonstration, BIO Web of Conferences 1 (2011) 00039.

43. https://www.youtube.com/watch? $\mathrm{v}=\mathrm{r} 7 \mathrm{gU} 74$ Yv9Es\&list $=$ UU6HrPPoLdjNynZCvUrJbmBw

44. S. Calinon, Challenges in adapting imitation and reinforcement learning to compliant robots, Bio Web of Conferences Skills 1 (2011) 00013.

45. R.N. Shea, Robot Safety standard Update, RIA meeting, 20-23 Feb 2013, Orlando World Marriot Centre, Orlando Florida.

46. http://www.electrothermindustry.com/Small\%20Rotary $\%$ 20 Hearth \%20Furnace

47. http://www.hse.gov.uk/statistics/industry/manufacturing/ manufacturing.pdf

48. Durosay et al., Journal of Hand Surgery (European) 36, 5, 383-391.

49. A. Razali, Y. Qin, J. Zhao, C.S. Harrison, R. Smith, Development of a new high precision feeder for micro-sheet forming, Journal of Manufacturing Science and Engineering, Transactions of the ASME 133, 6 (2011) 061025, DOI: 10.1115/ 1.4005046

50. Y. Qin, A. Brockett, Y. Ma, A. Razali, J. Zhao, C.S. Harrison, W. Pan, X. Dai, D. Loziak, Micro-manufacturing research, technology outcomes and development issues, International Journal of Advanced Manufacturing Technology 47, 9-12 (2009) 821-837, DOI: 10.1007/s00170-009-2411-2.

51. F.R. Spellman, Nancy E. Whiting, Machine Guarding Handbook, Rowan and Littlefield, 1999.

52. D.G. Sanders, Reinforced ceramic dies for superplastic forming operations, Journal of Materials Engineering and Performance 13, 6 (2004) 753-757.

53. http://www.forgex.co.uk 
54. http://www.CorePD.co.uk

55. www.strath.ac.uk/afrc

56. C.S. Harrison, R.J. Twist, K.K.B. Hon, Simulation of the effects of uncertainty and its effects on production planning lead times, in 6th International Conference on Computer Aided Production Engineering, University of Edinburgh, 1990, pp. 237-244.

57. A. Carrie, Simulation of Manufacturing Systems, John Wiley, 1988.

58. http://www.hse.gov.uk/pubns/books/1112.htm
59. http://www.fanucrobotics.co.uk/

60. S. Sakabara, The latest Robot systems which reinforce the manufacturing sector, in Proceedings of the 2003 IEEE International Conference on Robotics and Automation, Taipei, September (2003).

61. Z. Haiming, Y. Xichen, L. Lifeng, Path planning and off-line programming in laser remanufacturing robot system, Mechanic Automation and Control Engineering (MACE) (2010) 3796-3798.

Cite this article as: Harrison CS: A review of automation in manufacturing illustrated by a case study on mixed-mode hot forging. Manufacturing Rev. 2014, 1, 15. 